\title{
Image Processing Based Automatic Visual Inspection System for PCBs
}

\author{
Sanveer Singh ${ }^{1}$, Manu Bharti ${ }^{2}$ \\ ${ }^{I}$ (ECE Deptt., BGPC, Sangrur, India \\ ${ }^{2}$ (ECE Deptt., BGIET, Sangrur, India
}

\begin{abstract}
The on-line or automatic visual inspection of PCB is basically a very first examination before its electronic testing. This inspection consists of mainly missing or wrongly placed components in the PCB. If there is any missing electronic component then it is not so damaging the PCB. But if any of the component that can be placed only in one way and has been soldered in other way around, then the same will be damaged and there are chances that other components may also get damaged. To avoid this, an automatic visual inspection is in demand that may take care of the missing or wrongly placed electronic components.

In the presented paper work, an automatic machine vision system for inspection of PCBs for any missing component as compared with the standard one has been proposed. The system primarily consists of two parts: 1) the learning process, where the system is trained for the standard PCB, and 2) inspection process where the PCB under test is inspected for any missing component as compared with the standard one. The proposed system can be deployed on a manufacturing line with a much more affordable price comparing to other commercial inspection systems.
\end{abstract}

\section{INTRODUCTION}

The PCB (Printed Circuit Board) industry continues to adopt increasingly higher Windowing and Defect Extraction Operation levels of integration and achieving higher and higher levels of component density. As a consequence, the tolerances on PCB assembly become tighter and tighter. This causes an increased need for reliable and accurate visual inspection of PCB boards[1]. The manufacturing of PCB circuits uses the SMT (Surface Mount Technology). The SMT circuit assembly consists of three major processes, screen printing solder paste on the PCB, component placement and then solder re-flow in a convection oven. Correspondingly, there are three main tasks of vision inspection in PCB assembly:

Solder paste inspection,

Component placement, and

Post-reflow inspection.

Placing a proper amount of solder paste on a pad is the key to prevent unwanted opens or shorts. Sometimes, it is possible to catch these unwanted opens or shorts using an in-circuit-test after all components are placed on the board, but most solder paste defects are impossible to catch after components are mounted.

Presently, the PCBs are inspected for either the components inspection (missing or wrongly placed) or track inspection (broken). However, in the proposed system, it is expected to inspect the PCB under two layers:

Components Inspection (missing, wrongly placed or placed or wrong orientation)

Tracks Inspection (broken or shorting). This is called as bare Board Testing (BBT), on both sides in case of double side PCBs.

\section{BRIEF LITERATURE SURVEY}

Automatic optical inspection (AOI) systems are widely adopted in industrial applications [1-3]. In PCB inspection, AOI systems are used to detect surface-related defects such as bare-board inspection, solder bridging, lack of solder, missing components, poor part orientation, lifted leads, tombstoning, solder balls, and etc. [4]. Teoh et al. [5] used histogram to identify missing components or misalignment of component. They also proposed white pixel count index as a criterion to detect component with wrong orientation. Loh [6] proposed runlength encoding technique to detect a wrong integrate circuit component. Peng [7] used pattern matching to detect missing component. Most image features were only useful for detecting some specific types of defect for certain types of component. And, select proper image features and proper thresholds to detect various defects and classify these defects were not easy [8]. 
Image Processing Based Automatic Visual Inspection System for PCBs

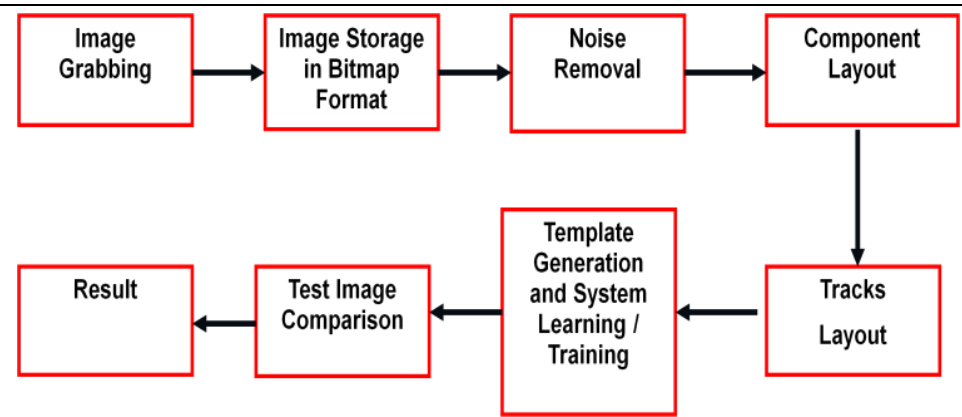

Block Diagram of the Proposed System

\section{ALGORITHM}

The image of the PCB is first grabbed using CCD camera and stored in 256 gray level shades in bitmapped format (BMP format). The noise present in terms of scattered pixels is removed and components are identified with respect to their location, size and count. The set of data so obtained is used as reference or standard data for comparison purposes. The PCB under test is subjected to same process. The new set of data is computed from the grabbed image of test PCB and compared with the standard data. The decision is made on the basis of comparison of two data.

The system's intelligence is made function of the quality of standard data computed from the standard PCB. More is the information computed form the standard image, more productive result could be deduced. This is the key factor behind the machine vision system for PCBs inspection. Otsu Algorithm is used for image thresholding. This works fine for PCB image enhancement. Salt and Pepper noise is removed median filtering. Tracks information with respect to their location and dimension. A data file is generated for each PCB including Ref. (Standard) PCB and and Test PCB. On comparison of both data file, result is deduced. Fig. 1 and 2 shows the PCB images before and after thresholding.

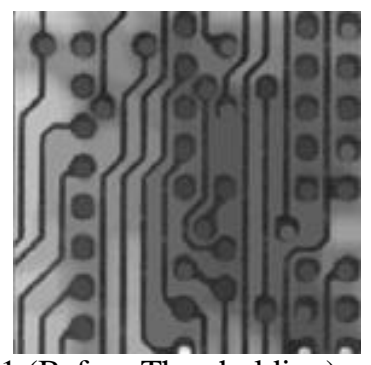

Fig. 1 (Before Thresholding)

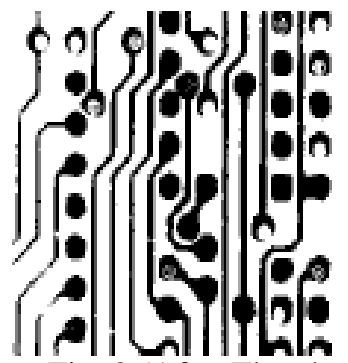

Fig. 2 (After Thresholding)

IV. NOISE REMOVAL

Salt and Pepper noise are removed by applying the following algorithm:

If $(\mathrm{P} 0=\mathrm{BLACK}) \& \mathrm{P} 1=\mathrm{P} 2=\mathrm{P} 3=\mathrm{P} 4=\mathrm{P} 5 \mathrm{P} 6=\mathrm{P} 7=\mathrm{P} 8=$ WHITE $)$

Then $\mathrm{P} 0$ is the Background Pixel.

If $(\mathrm{P} 0=$ WHITE $) \& \mathrm{P} 1=\mathrm{P} 2=\mathrm{P} 3=\mathrm{P} 4=\mathrm{P} 5 \mathrm{P} 6=\mathrm{P} 7=\mathrm{P} 8=\mathrm{BLACK})$

Then $\quad \mathrm{P} 0$ is the Object Pixel.

The intensity values of pixels in a small region within the size of the filter are examined, and the median intensity value is selected for the central pixel. Removing noise using the median filter does not reduce the difference in brightness of images, since the intensity values of the filtered image are taken from the original image.

\section{Feature Extraction Process}

\begin{tabular}{|l|l|l|}
\hline $\mathrm{P}_{8}$ & $\mathrm{P}_{1}$ & $\mathrm{P}_{2}$ \\
\hline $\mathrm{P}_{7}$ & $\mathrm{P}_{0}$ & $\mathrm{P}_{3}$ \\
\hline $\mathrm{P}_{6}$ & $\mathrm{P}_{5}$ & $\mathrm{P}_{4}$ \\
\hline
\end{tabular}


Feature extraction process consists of four steps:

Coarse resolution pcb defect localization algorithm

Window coordinates searching operation

Mapping operation.

Windowing and defect extraction operation

The defect localization is important in order to mention the users about the location of the defects detected for further procedures such as defect classification and defect marking. The input for the defect localization algorithm is the coarse differenced image. The defect localization algorithm is based on connectedcomponent Labelling Operation. For a two dimensional array, there exist two types of connectivity. The first one is 4-connected pixel and the second one is 8-connected pixel. The connected-component labelling operation returns the information of the coarse differenced image (a binary image) to identify each object in the image. The output of the connected-component labelling operation is a two dimensional output array named as labelled image. In matlab, this operation is performed using bwlabel command.

The labeled image is input for the window coordinate searching operation. The objective of this operation is to search four coordinates of each object in the defective area window.

According to the coordinates obtained in window coordinate searching operation, a number of windows are drawn on the fine resolution tested image. The determination of the mapping equation is critical in the sense that ineffective mapping equation will cause the distortion problem happened to the individual drawn window.

For pixels related to respective defective area, a boundary line representing a defective window can be drawn on the fine resolution tested PCB image. Each window marks the defective areas where the defects are actually occurred. After the defective areas are windowed successfully, it is possible to segment each defective area for defect extraction where each defective area is shown in an individual image

\section{RESULTS}

Component Location is identified by its starting pixel and a rectangle is marked around its periphery. In below fig. 3 and 4, rectangular marks are shown.

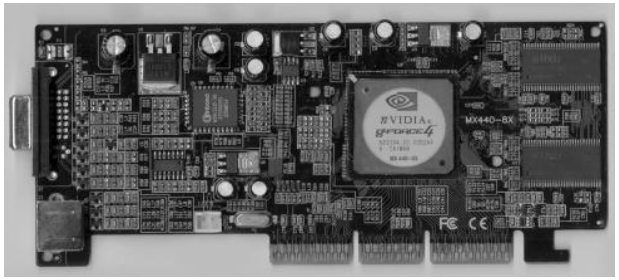

Fig. 3

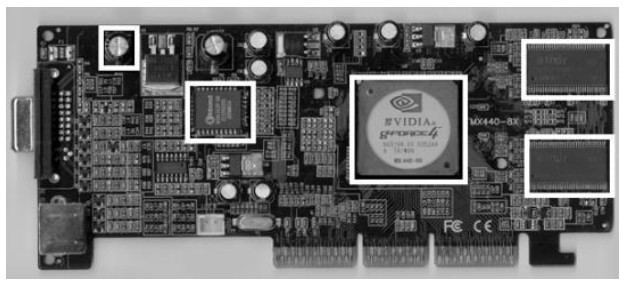

Fig. 4

In below figures fig. 5 and 6 , track missing area is identified. When these tow images are subtracted from each other, fig. 7 is obtained. 


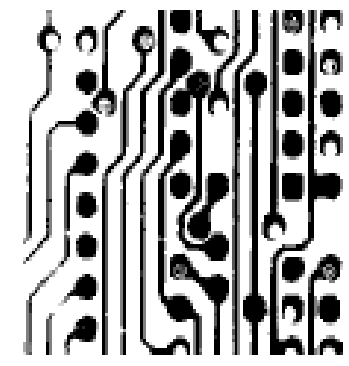

Fig. 5

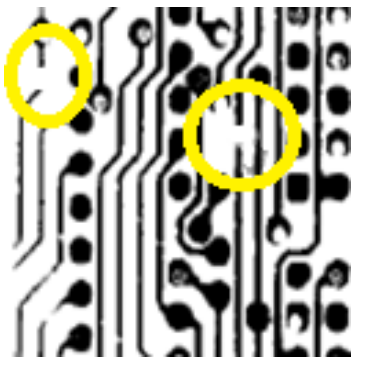

Fig. 6

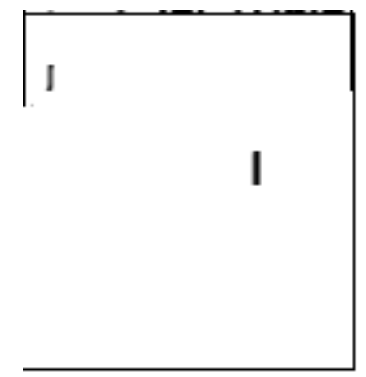

Fig. 7 (Subtracted Image)

The two tracks shown in fig. 7 are the result of the standard image and test image. Tracks are tested by just taking the one to one difference of the two PCBs Images.

$R=f(s)-f(t)$

Where $\mathrm{f}(\mathrm{s})$ and $\mathrm{f}(\mathrm{t})$ are the tracks pixel location vectors of standard and test PCBs.

The wrongly placed component (IC) is identified by its slot identification. If the slot location is not matched with the standard PCB slot location, this shows that the IC is placed in wrong orientation.

Finally on the basis of comparison of the two PCBs, i.e. Standard PCB and PCB under test, result is deduced for its

OK/Not OK Report The comparison result $\mathrm{R}$, is computed by difference of the two templates.

$$
R=f(s)-f(t)
$$

Where $\mathrm{f}(\mathrm{s})$ and $\mathrm{f}(\mathrm{t})$ are the component location vectors of standard and test PCBs.

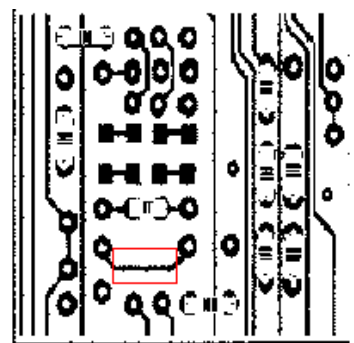

Fig. 8

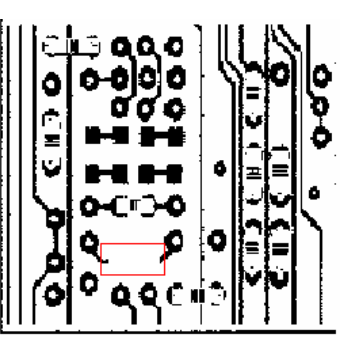

Fig. 9

VII. CONCLUSION

The proposed system is not the generalized system but a dedicated system for a particular PCB and can be optimized to a level. This is also important to note that the system can not be generalized as each PCB inspection is different from its assembly point of view. Above all, it's NDT testing i.e. Non-destructive testing and the PCBs need not to be given any power supply for its test under machine vision system.

\section{ACKNOWLEDGEMENTS}

We are thankful to Mr. Vikas Goel, Sr. Project Manager, C-DAC, Mohali, Punjab for his valuable guidance and continuous support in making this paper. 


\section{REFERENCES}

[1] M. Moganti, F. Ercal, "Automatic PCB inspection Understanding, vol. 63, no. 2, pp. 287-313, 1996.

[2] E. K. Teoh, D. P. Mital, B. W. Lee, and L. K. Wee, '90., 16th Annual Conference of

"Automated Visual Inspection of Surface Mount

PCBs, " IECON

IEEE, pp. 27-30, November. 1990. Applications, vol. 35, no. 2, pp. 426-432, 1999.

[4] D. B. Perng, G. Y. Peng, C. J. Yan, and C. C. Chou, "A new machine vision system for SMD PCB auto-inspection," The International

Symposium on Measurement Technology \& $\quad$ Intelligent Instrument ISMTII' 2001, Cairo, Egypt, 25 - 29 Sept. 2001.

[5] Y.H. Lin, "The estimation for algorithms of visual inspection of surface mount PCBs," Master Thesis, National Tsing Hua University, Taiwan, 2003.

[6] D. L. Tsai, C. H. Tsai and S. C. Lin, "Using neural network for computer vision inspection of printed circuit board," Proc. 4th Conference on Precision Machinery and Manufacturing, A03-004, Taipei, 2004.

[7] L. V. Fausett, Fundamentals of neural networks, Prentice-Hall, Englewood, Cliffs, NJ, 1994.

[8] K. Tarun, and T. Khanna, Foundations of neural networks, Reading, Mass. Addison-Wisley, 1990.

[9] P. Piction, Introduction to neural networks, London: Macillan, 1994. 